\title{
Discussing Sudden Unexpected Death in Epilepsy (SUDEP) with Patients: Practices of Health-Care Providers
}

\author{
Wendy R. Miller, PhD, RN, CCRN, \\ Assistant Professor, Department of Science of Nursing Care Indiana University School of Nursing \\ 1033 East Third Street Bloomington, IN 47405 812-855-4971 wrtruebl@iu.edu
}

\author{
Neicole Young, BSN, RN, \\ Doctoral Student Indiana University School of Nursing 1111 Middle Drive Indianapolis, IN 46202 \\ nyoung28@iupui.edu
}

Daniel Friedman, MD,

Assistant Professor, Department of Neurology NYU School of Medicine 223 East $34^{\text {th }}$ Street New

York, NY 10016 daniel.friedman@nyumc.org

Janice M. Buelow, PhD, RN, FAAN, and

Vice President of Programs and Research Epilepsy Foundation of America 8301 Professional

Place Landover, MD 20785 jbuelow@efa.org

Orin Devinsky, MD

Professor, Department of Neurology NYU School of Medicine 223 East 34 ${ }^{\text {th }}$ Street New York, NY 10016 od4@nyu.edu

\section{Introduction}

Sudden unexpected death in epilepsy (SUDEP) is the most common disease-related cause of death in persons with epilepsy. SUDEP includes all epilepsy-related deaths not due to trauma, drowning, status epilepticus, or other identifiable causes [1]. Evidence from epidemiologic, observational, clinical, and pathological studies strongly suggests that SUDEP occurs after generalized tonic-clonic seizures [2].

The physiologic mechanisms underlying SUDEP are poorly understood [2]. However, based on the literature, several authors have suggested potential strategies to prevent SUDEP: good seizure control, reduction of stress, participation in physical activity, supervision at night, seizure monitors that can detect motion or other physiological parameters, and family training in CPR [2, 3]. Self- and family-management of epilepsy is critical to seizure control. Therefore, patients and families should be knowledgeable about SUDEP and the importance of seizure control in potentially preventing SUDEP.

Professional organizations, including the American Epilepsy Society [4], National Institute for Health and Care Excellence [5], and the Scottish Intercollegiate Guidelines Network [6], espouse the practice of discussing SUDEP with patients and family members, yet do not provide clear guidelines in terms of how—or when—-these discussions should take place.

(C) 2013 Elsevier Inc. All rights reserved.

Correspondence to: Wendy R. Miller.

Publisher's Disclaimer: This is a PDF file of an unedited manuscript that has been accepted for publication. As a service to our customers we are providing this early version of the manuscript. The manuscript will undergo copyediting, typesetting, and review of the resulting proof before it is published in its final citable form. Please note that during the production process errors may be discovered which could affect the content, and all legal disclaimers that apply to the journal pertain. 
Consensus is lacking on how health care practitioners should address SUDEP with patients and family members, and evidence regarding patient educational interventions is lacking as well. In general, physicians have employed a physicianled, rather than a patient-centered, approach to addressing SUDEP with patients. A study examining "truth telling" practices among Italian epileptologists found that $61.8 \%$ of the sample discussed SUDEP with very few of their patients [7]. Another study explored the effect of talking to patients about SUDEP. When parents were told about the potential of SUDEP in their children, their initial distress did not continue. Children in the study were rarely told about SUDEP [7, 8]. An indepth investigation into the SUDEP-discussing practices of a variety of practitioners who commonly provide care to persons with epilepsy is needed. Specifically, uncovering providers' reasons for discussing or not discussing SUDEP with patients and family members is needed to allow for a more informed establishment of SUDEP-discussing practices.

The purpose of this study was to describe the practices of epileptologists, neurologists, and advanced practice nurses (APNs) regarding discussing SUDEP with their patients, as well as to reveal the rationale for their SUDEP-discussing practices; this purpose was fulfilled using focus group methods and thematic analysis.

\section{Methods}

\subsection{Participant recruitment}

The study was approved by the Indiana University Institutional Review Board. Between December 2011 and September 2012, we recruited adult and pediatric epileptologists, neurologists, and advanced practice nurses (APNs) at national multi-disciplinary meetings of neurology or epilepsy providers (American Epilepsy Society and the American Association of Neuroscience Nurses) to capture the perspective of various epilepsy health care providers. Invitations to take part in the study were distributed at multiple education venues at these meetings. Email blasts were also used to alert providers of the opportunity to participate. Therefore, all registered meeting attendees had the opportunity to participate. Providers interested in participating in the study contacted the researchers.

\subsection{Data collection}

All data were collected via focus groups [9], and separate focus groups were held for each of the three categories of providers. Five separate focus groups (all of which included a mix of pediatric and adult practitioners) were conducted - two for epileptologists, two for neurologists, and one for APNs. Prior to the initiation of each focus group, demographic data were collected. Each of the three focus groups was moderated by one of the study investigators. The moderator asked participants questions pertaining to reasons for and for not discussing SUDEP, and how they discuss SUDEP (see Appendix). Follow-up questions were asked based on discussion between the participants. Focus groups ranged from 60 to 90 minutes in duration and were audio recorded. Audio recordings were then transcribed verbatim.

\subsection{Data analysis}

All transcripts were subjected to conventional content analysis [10]. Sandelowski's [11] initial steps for the handling of qualitative data were used as a starting point for analysis. Data were analyzed per each provider group, and codes were generated; codes were organized according to main questions asked and discussed during the focus groups. Next, comparisons of codes were made across groups. Data from all groups were then organized via themes in order to fulfill the purpose of the study. 


\section{Results}

\subsection{Sample}

A total of 43 providers, including 19 epileptologists, 16 neurologists, and 8 APNs, participated. Of the epileptologists and neurologists, eight were female, and all of the APNs were female. While we did not collect extensive demographic data, the west coast, east coast, and central areas of the United States were all represented, and only four participants had fewer than five years of experience.

\subsection{Themes}

Themes were generated for the following areas of interest: reasons for discussing SUDEP (five themes), reasons for not discussing SUDEP (three themes), and ways in which SUDEP should be discussed (three themes).

3.1.1. Reasons for discussing SUDEP-Across all disciplines, reasons for discussing SUDEP included: Practical Accountability, Moral Accountability, Proactivity, and Reactivity. For nurses only, an additional reason was Patient Advocacy.

3.1.1.1. Practical Accountability: Practical accountability refers to a provider offering patients and family members accurate SUDEP information to facilitate informed decisionmaking. All three types of providers stated that they sometimes discuss SUDEP with patients or family members due to feeling practically accountable to them. The following selections from answers to questions and general discussion in the focus groups illustrate practical accountability.

1. "We talk about things like accidents that can occur, injuries that can occur, not driving, fatalities that can result from driving. I think SUDEP should be just part of that practical discussion." (Epileptologist)

2. "We inform every one of them that there are possibilities of complications that might include sudden death, with or without an accompanying seizure...so they have that information to base decisions on." (Neurologist)

3. 'You talk about well, you're already concerned and nervous about this when you react to-about SUDEP. You are already worried so let's talk about what you can do. What can you do to try to prevent this. Give them some information to use." (APN)

3.1.1.2. Moral Accountability: Moral accountability refers to discussing SUDEP with patients and family members in order to avoid withholding important, epilepsy-related information. Once again, all types of providers reported feeling a moral obligation to provide patients or their family members with information about SUDEP.

1. "If we don't share, as a physician that you know—-kind of you're taking responsibility." (Epileptologist)

2. "At some point in time, a conversation has to take place about what the end of life will be. Don't want caregivers to spend the rest of their life with guilt if they come into the nursery one morning and find the child blue and cold." (Neurologist)

3. "You don't want to put up fear, but it is something that does have to be discussed... or we aren't doing our jobs." (APN)

3.1.1.3. Proactivity: Proactivity refers to provider-initiated discussion of SUDEP to ensure that patients and family members receive accurate information about SUDEP, and to act as a 
filter against inaccurate information that patients or family might encounter elsewhere. All providers reported discussing SUDEP for this reason.

1. "I've moved toward trying in the first visit, or one of the first visits, because actually one of my concerns is that the educated patients are gonna go read about it somewhere else, and be upset that they hadn't had the discussion." (Epileptologist)

2. "I would do it early on, rather than them finding it on the Internet, and then coming in talking to me." (Neurologist)

3. "I think preventative it's an important discussion to have and approaching it from a safety standpoint." (APN)

3.1.1.4. Reactivity: Reactivity refers to a discussion of SUDEP triggered by a patient's behavior-noncompliance, a patient leaving home for the first time, for example. Members of all three provider groups reported discussions during which they would bring up SUDEP only after a patient did or was about to do something that the provider felt put the patient at higher risk of SUDEP.

1. 'I only discuss it in those settings where I felt that people weren't being compliant." (Epileptologist)

2. 'I'm not comfortable with every family to discuss [SUDEP]. I'd rather put it as aI would say as a weapon when they are not agreeing with the medications." (Epileptologist)

3. "Generally, I don't talk...about it unless-the older patients are really noncompliant with medications. I tend to worry about them and start talking to them about all the possibilities." (Neurologist)

4. "I make sure that any of my kids who are teenagers are going away to college- that I have that discussion...they're not under the parents' vigilant eyes." (APN)

3.1.1.5. Patient Advocacy: The APNs in the sample were the only group to report that they discuss SUDEP as patient advocates. That is, they feel that patients and family members have a right and a need to know about SUDEP and will initiate the discussion to promote the best interest of the patient or family.

1. 'What'll happen is I'll be bringing it up with a patient that seems appropriate...a lot of times these patients will've been seen by our neurology division for a long time and no one's ever brought it up...they'll say 'Well, I've been seeing doctor soand-so and no one's ever mentioned this as a possibility."' (APN).

2. "I think there are ways of talking about it. I mean, after all we talk about not driving and the risks of driving with epilepsy. We seem much more comfortable talking about the risk of driving with uncontrolled seizures than we do about the risks of the disease itself." (APN).

3. "[I discuss it] to have parents recognize that this can happen and precautions need to be taken." (APN).

3.1.2. Reasons for not discussing SUDEP-In terms of when not to discuss SUDEP, for all types of providers involved, and especially the physicians, the theme Not at First emerged. Additional themes that emerged for this question included, in the case of neurologists and epileptologists, Moral Accountability and Out of Options. 
3.1.2.1. Not at First: Members of all three provider groups indicated that, in some cases, they find it best to wait to discuss SUDEP with patients and families until a rapport has been established.

1. "For me, to talk about it at that first appointment, and it mostly has to do with practice style. That's an encounter where the patient's meeting you for the first time. They want to feel that they're going to be better. They want to feel that they can trust the doctor in terms of making them better." (Epileptologist)

2. "Sometimes parents or even patients from the first time, they ask you"Is my child going to die from this?" We have to discuss it at that point, but I'm not sure if we need really to scare them from the first time we meet them." (Neurologist)

3. "It's not always good to scare them right away in the middle of this huge diagnosis." (APN)

3.1.2.2. Moral Accountability: Both epileptologists and neurologists cited moral accountability as a reason not to discuss SUDEP (just as they, along with APNs, also cited such accountability as a reason to discuss SUDEP). Physicians explained that they sometimes choose not to discuss SUDEP with patients and family because they feel it is morally wrong to give information about a complication that is poorly understood and difficult to prevent.

1. "In the case of SUDEP, to alarm somebody unnecessarily is concerning. If there's a purpose-if you can benefit the patient then that's a different matter. I think that to me the key is how will this benefit the patient versus unnecessarily alarming the patient and the family and alerting them to something that we really can't do anything about." (Epileptologist).

2. "You have to consider will this just serve to make them more anxious, or can it actually help?" (Neurologist)

3.1.2.3. Out of Options: Both physician groups indicated that they often choose not to discuss SUDEP when all epilepsy treatment options have been attempted and have failed.

1. 'I have patients who are - I don't have anything else to offer them, and I know that they are at risk, and I know they're very anxious about everything in life, and often sometimes a little bit simple. I don't see the point in giving them something else. There's nothing they can do about it. I do feel conflicted about that, and may change my practices, but it's very hard." (Epileptologist)

2. "When there is no response to treatment and nowhere else to go, if it is very severe, I don't always discuss [SUDEP]." (Neurologist)

3.1.3. Ways in which to discuss SUDEP-Ways in which SUDEP is discussed included, in all provider groups, Discussion and Written Materials. In addition, prevalent in all groups was the finding that procedures for discussing SUDEP with patients and families need to be somewhat standardized, though the discussion should always be tailored to fit the patient's context. As well, all three types of providers expressed the idea that more informative written materials regarding SUDEP should be developed.

3.1.3.1. Discussion: Providers from all three groups indicated that they frequently use a face-to-face unscripted format for SUDEP discussions. 
1. "When it comes up [in clinic] it's not a very long conversation but as I'm talking about other things I mention it and that I don't exactly know how their epilepsy is going to progress but we talk about consequences." (Epileptologist)

2. "In my experience it just comes up sometimes in the clinic, and I answer questions." (Neurologist)

3. "We sit down and we talk with them and answer questions right there." (APN)

3.1.3.2. Written Materials: Members of both physician groups, as well as APNs, report providing patients and family members with written informational materials pertaining to SUDEP. Most reported providing patients and families with materials developed by the Epilepsy Foundation.

1. "The Epilepsy Foundation has educational materials, and we give patients access to those." (Epileptologist)

2. "We typically use the [Epilepsy Foundation] materials as handouts after the discussion." (Neurologist)

3. "Often times we provide them with education pamphlets from [The Epilepsy Foundation]." (APN)

3.1.3.3. Standardization: All three types of providers felt that the process for discussing SUDEP with patients and family members should be somewhat standardized. However, they also indicated that SUDEP discussions should be tailored to patients' and families' needs. In addition, all three groups were somewhat unsatisfied with current SUDEP educational materials available for distribution to patients and family members, and felt that a need exists for the development of more appealing and accurate SUDEP materials.

1. "So you give them the pamphlet...which is in my mind, is the low level. They'll look at it; they'll toss it; they'll lose it...there needs to be some improvements made to the materials, and they should be standardized." (Epileptologist)

2. "I think we all do different things, what we are comfortable with. As a profession we probably need more guidance in terms of exactly what to cover, and maybe how. But you always have to consider that person's situation...their anxiety, their risk, all that." (Neurologist)

3. "What we have to offer them might not be helpful to everyone... it would be good to revisit those materials and improve them." (APN)

\section{Discussion}

Our focus groups of providers caring for epilepsy patients revealed that their decisions about discussing SUDEP with patients and families were strongly influenced by personal views that can be understood in the context of thematic approaches. The key elements of those who discuss SUDEP included practical and moral accountability, proactivity and reactivity to patient decisions about self-management, and advocacy (APN only). The key elements in decisions not to discuss SUDEP included a delay in the conversation until the patientprovider relationship and trust is established and moral accountability of avoiding harm if there is little or no perceived benefit to having the discussion. Practical and moral accountability described the providers' rationale for discussion, whereas proactive and reactive discussions were meant to affect certain behaviors. Providers made decisions about SUDEP discussion based on what they believed would be best for the patient. 
While all of the providers reported having given thought to discussing SUDEP with their patients, many reported being reticent to initiate such a discussion. The vast majority of patients want information about SUDEP from their neurologists and epileptologists [8]. Yet, in many cases, these practitioners do not routinely, and in some cases, rarely, discuss SUDEP [12]. The consequence of providers' hesitance to discuss SUDEP is that patients and their family members are uninformed about the possibility of sudden death due to epilepsy, whether from SUDEP or other causes of epilepsy-related mortality (eg., status epilepticus, accident, drowning, suicide). For example, several medical examiners told us that they avoid SUDEP on death certificates since they have occasionally had families who are adamant that their neurologists told them that seizures are never fatal (O. Devinsky, personal communication, June, 2013). The gap between what patients want to know and what information neurologists, epileptologists, and APNs will routinely provide is significant. The decision-making strategies of the providers in this study, while extremely well-intended, were not necessarily patient-centered. They did not involve patients in the decision-making process. This study highlighted key thematic issues that help account for this gap and suggests approaches to help narrow it.

Perhaps the most effective way to encourage providers caring for epilepsy patients to more fully discuss epilepsy-related injuries and mortalities, including SUDEP, is to build on the existing foundation. Since all providers seek to improve their patients' health through education, accurate diagnosis and effective therapy, SUDEP education should be a component of epilepsy care.

Practitioners should more actively include patients in the decision-making process and provide patients with the necessary information to make sound decisions about their own care (13). This study's themes of practical and moral accountability partly address patientcentered care since providers recognize the importance of providing information about SUDEP. Yet those themes also inhibited practitioners from talking to their patients about SUDEP if they believed that informing patients might do harm. All providers should be educated about the importance of informing patients and families about SUDEP targeting these thematic issues and including a more patient-centered approach. SUDEP should be part of basic epilepsy education and care (practical accountability). Educating patients about SUDEP may reduce the morbidity and mortality of epilepsy by improving compliance, modification of lifestyle factors and other safety measures by providing information upon which patients can make self-management decisions. It is ethical care to improve the potential health outcomes (moral accountability). Accurate information can help prevent unnecessary fears among many patients and families and replace misinformation from unreliable sources (proactivity). While reactivity is a common strategy and often triggered by a breakthrough seizure or patient admitting to a period of nonadherence or unhealthy lifestyle choices (e.g., sleep deprivation, excess alcohol), education should be employed to prevent these events. However, a breakthrough seizure or change in lifestyle that increases seizures may be an opportunity to inform patients about the importance of seizure freedom. Patients and families want to know about SUDEP and should be informed (advocacy).

This study provided an initial description of health care providers' practices in SUDEP discussion. We were able to identify thematic approaches to SUDEP discussion and specific reasons for why health care providers do or do not talk about SUDEP. Our goal is to help providers become adept at initiating this difficult discussion with patients, thus building a partnership to enhance epilepsy management. Because health care providers clearly believe it is their practical and moral responsibility and want to be proactive in helping patients to achieve their goals and advocate for patients, the next step is to provide a practical guide to a discussion of SUDEP. Potential limitations to the generalizability of findings from this study include a lack of care providers from outside the United States, and that care providers who 
chose to participate in the study may be significantly different (in terms of SUDEPdiscussing practices) from those who elected not to take part.

\section{Acknowledgments}

We wish to acknowledge Paul Buelow, $\mathrm{PhD}$ for his efforts in editing this manuscript.

This work was supported in part by T32NR007066 and KL2TR000163, both of the National Institutes of Health

\section{References}

1. Nashef L, So EL, Ryvlin P, Tomson T. Unifying the definitions of sudden unexpected death in epilepsy. Epilepsia. 2012; 53(2):227-233. [PubMed: 22191982]

2. Devinsky O. Sudden, unexpected death in epilepsy. N Engl J Med. 2011; 365:1801-1811. [PubMed: 22070477]

3. Scorza FA, Arida RM, Terra VC, Cavelheiro EA. What can be done to reduce the risk of SUDEP? Epilepsy Behav. 2010; 18:137-138. [PubMed: 20537595]

4. American Epilepsy Society. Support for increased awareness of SUDEP. Hartford (CT): [Internet]. [updated, 2013, cited 2013 Oct 29]. Available from: http://www.aesnet.org/pressroom/consensusstatements/support-for-increased-awareness-of-sudep

5. National Institute for Health and Care Excellence. The epilepsies: the diagnosis and management of the epilepsies in adults and children in primary and secondary care. London (UK): [Internet]. [updated, 2012, cited 2013 Oct 29]. Available from: http://www.nice.org.uk/cg137

6. Scottish Intercollegiate Guidelines Network. Diagnosis and management of epilepsy in adults. Edinburgh (UK): [updated, 2005, cited 2013 Oct 29]. Available from: http://www.sign.ac.uk/ guidelines/fulltext/70/index.html

7. Vegni E, Leone D, Canevini MP, Tinuper P, Moja EA. Sudden unexpected death in epilepsy (SUDEP): a pilot study on truth telling among Italian epileptologists. Neurol Sci. 2011; 32(2):331335. [PubMed: 20607335]

8. Gayatri NA, Morrall MC, Jain V, Kashyape P, Pysden K, Ferrie C. Parental and physician beliefs regarding the provision and content of written sudden unexpected death in epilepsy (SUDEP) information. Epilepsia. 2010; 51(5):777-782. [PubMed: 20067505]

9. Patton, M. Qualitative research and evaluation methods. 3rd ed. California: Sage; 2001.

10. Hsieh H, Shannon S. Three approaches to qualitative content analysis. Qual Health Res. 2005; 15(9):1277-1288. [PubMed: 16204405]

11. Sandelowski M. Qualitative analysis: what it is and how to begin. Res Nurs Health. 1995; 18(4): 371-375. [PubMed: 7624531]

12. Morton B, Richardson A, Duncan S. Sudden unexpected death in epilepsy: Don't ask? Don't tell? J Neurol Neurosurg Psychiatry. 1996; 77:199-202. [PubMed: 16421121]

13. Coelho T. A patient advocate's perspective on patient-centered comparative effectiveness research. Health Aff. 2010; 29:1885-1890.

\section{Appendix}

Question Guide used in Focus Groups*

a. Do you discuss SUDEP with all of your patients and their families? Why or why not?

b. When you do discuss SUDEP with patients or families, how do you structure that discussion?

c. When you do discuss SUDEP, what content or information do you include?

d. When do you feel it is most important to discuss SUDEP? When is it least important? 
e. How do you think practitioners should approach SUDEP discussion with patients and their family members?

f. Do you feel that your patients and their families want to know about SUDEP? Why or why not?

*These questions were used as a starting point for each focus group. Questions were added based on participants' responses to initial questions and dialogue with each other. 


\section{Highlights}

- We examine SUDEP-discussing practices of epilepsy providers.

- Provider decisions about discussing SUDEP are influenced by personal views.

- Key themes are moral/practical accountability and reactivity/proactivity.

- A practical, patient-centered guide to the discussion of SUDEP is needed. 